\title{
Measurement of photon production cross sections with the ATLAS detector
}

\author{
Ruggero Turra, on behalf of the ATLAS Collaboration.* \\ INFN Sezione di Milano, Milan, Italy \\ E-mail: ruggero.turra@mi.infn.it
}

\begin{abstract}
The production of prompt isolated photons at hadron colliders provides a stringent test of perturbative QCD and can be used to probe the proton structure. The ATLAS Collaboration [1] has performed precise measurements of the inclusive production of isolated prompt photons at a centre-of-mass energy of $13 \mathrm{TeV}$, differential in both rapidity and photon transverse momentum. In addition, the integrated and differential cross sections for isolated photon pairs at $8 \mathrm{TeV}$ have been measured. The results are compared with state-of-the-art theory predictions at NLO in QCD and with predictions of several MC generators.

The production of prompt photons in association with jets provides an additional testing ground for perturbative QCD (pQCD) with a hard colourless probe less affected by hadronisation effects than jet production. The dynamics of isolated-photon plus jet production in pp collisions at a centre-of-mass energy of 8 and $13 \mathrm{TeV}$ will be presented and discussed.
\end{abstract}

The European Physical Society Conference on High Energy Physics

5-12 July, 2017

Venice

${ }^{*}$ Speaker. 
The production of prompt photons in proton-proton collisions, provides a test for perturbative QCD (pQCD) with a hard colourless probe and it is sensitive to the parton density function (PDF) of the gluon. All photons produced in pp collisions that are not secondaries from hadron decays are considered as "prompt". Comparisons of prompt-photon data and PQCD are usually limited by the theoretical uncertainties associated with the missing higher-order terms in the perturbative expansion. To make such a test with small experimental and theoretical uncertainties, it is optimal to perform measurements of prompt-photon production at high photon transverse energies and at the highest possible centre-of-mass energy of the colliding particles.

Photons are reconstructed from the energy deposited in the electromagnetic calorimeter (ECAL). The photon identification selection is based on the energy fraction leaking into the hadronic calorimeter, and on the shower shape measured in the ECAL.

All the analyses require photons to be in the central part of ECAL, $|\eta|<2.37$ except $1.37<$ $|\eta|<1.56$, photon identification and a cut on the isolation energy measured by the calorimeter. The background, mainly from misidentified jets, is removed with data-driven techniques. Cross sections with inclusive isolated-photon [2], photon plus jets [3] at $13 \mathrm{TeV}$ and diphoton [5] at 8 $\mathrm{TeV}$ are presented. Usually the experimental uncertainties are smaller than the theoretical ones.

In the inclusive photon analysis at $13 \mathrm{TeV}$, photons with $E_{T}^{\gamma}>125 \mathrm{GeV}$ are used to measure the inclusive cross section as a function of the photon transverse energy $E_{T}^{\gamma}$ in four regions of photon pseudorapidity. The measurements are compared with Jetphox at NLO, Sherpa and Pythia Monte Carlo. The main experimental uncertainties come from the photon energy scale, photon identification and the background subtraction procedure. The theoretical uncertainties are dominated by the terms beyond NLO, from which it is concluded that NNLO pQCD corrections are needed to make an even more stringent test of the theory. Figure 1 shows the comparison between data and the predictions.

In the photon-jet analysis at $13 \mathrm{TeV}$ events are required to have a photon with $E_{T}^{\gamma}>125 \mathrm{GeV}$ and a jet reconstructed with anti- $k_{T}(R=0.4)$ with $p_{T}>100 \mathrm{GeV},|y|<2.37$, with an angular separation from the photon $\Delta R>0.8$. The measurements of angular correlations between the photon and the jet can be used to probe the dynamics of the hard-scattering process. Differential cross sections as a function of kinematic variables are studied: $\cos \theta^{*}=\tanh (\Delta y / 2)$ where $\Delta y$ is the difference between the rapidities of the two final-state particles, leading-photon transverse energy $\left(E_{T}^{\gamma}\right)$ and leading-jet transverse momentum $\left(p_{T}^{\text {jet-lead }}\right)$, angular separation in azimuth between the leading photon and the leading jet $\left(\Delta \phi^{\gamma-\mathrm{jet}}\right)$ and the invariant mass of the leading photon and the leading jet $\left(m^{\gamma-\text { jet }}\right)$. In particular $\cos \theta^{*}$ provides a handle on the relative contributions of the direct and fragmentation components as well as the possibility to test the dominance of t-channel quark exchange. The measurements are compared to next-to-leading-order (NLO) QCD predictions from Jetphox and Sherpa as well as to the tree-level predictions of Pythia and Sherpa. Some example are shown in Figure 2. In particular Sherpa is able to reproduce the data down to $\Delta \phi^{\gamma-\text { jet }}=\pi / 2$ due to the inclusion of the matrix elements for $2 \rightarrow n$ processes with $n=4$ and 5 . The measured dependence on $\left|\cos \theta^{*}\right|$ is consistent with the dominance of processes in which a quark is being exchanged.

Diphoton cross sections are sensitive to $\alpha_{s}$ correction, QCD infrared emission and New Physics. In the diphoton analysis at $8 \mathrm{TeV}$ two photons are required to have $E_{T}>40 \mathrm{GeV}$ or $30 \mathrm{GeV}$ and an angular separation $\Delta R>0.4$. The suppression of backgrounds allow to reach a purity around $75 \%$. 

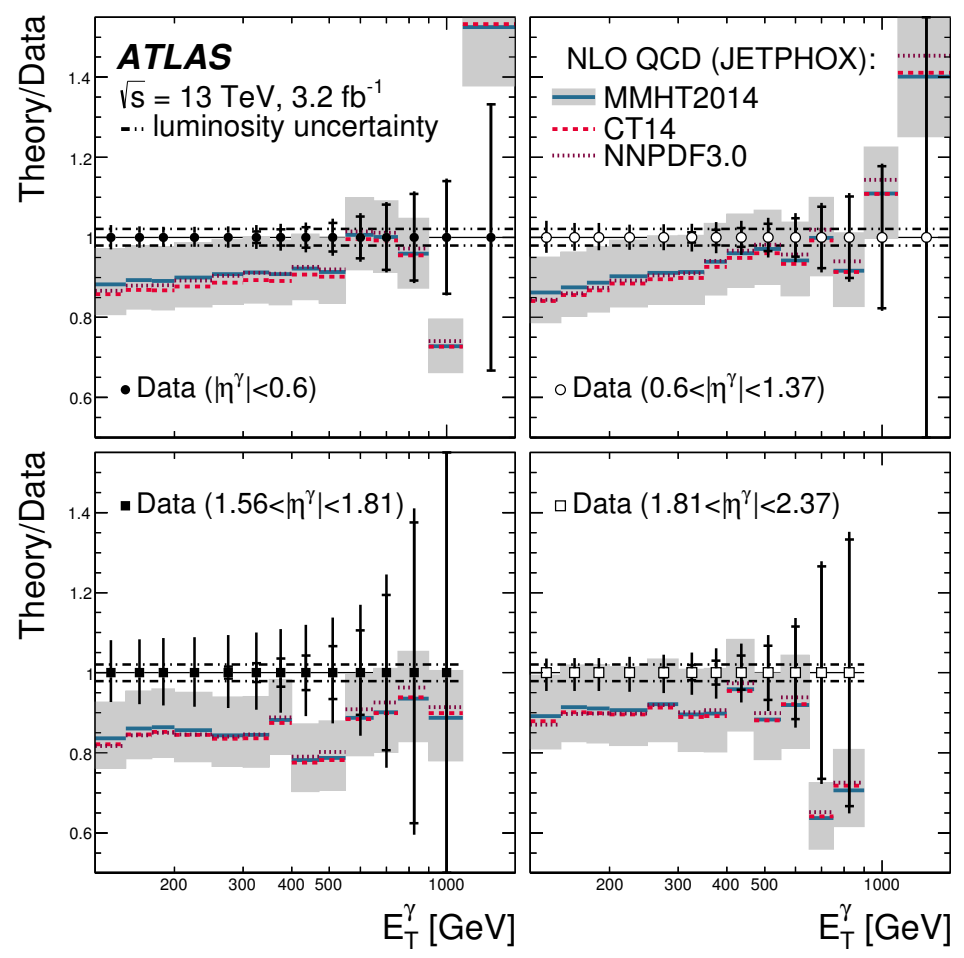

Figure 1: Ratio of the NLO pQCD predictions from Jetphox based on the MMHT2014 PDFs to the measured cross sections for isolated-photon production (solid lines) as a function of $E_{T}^{\gamma}$ in four bins of pseudorapidity. The ratio of the NLO pQCD predictions based on the CT14 (dashed lines) or NNPDF3.0 (dotted lines) PDF sets to the data are also included. Ref. [2].
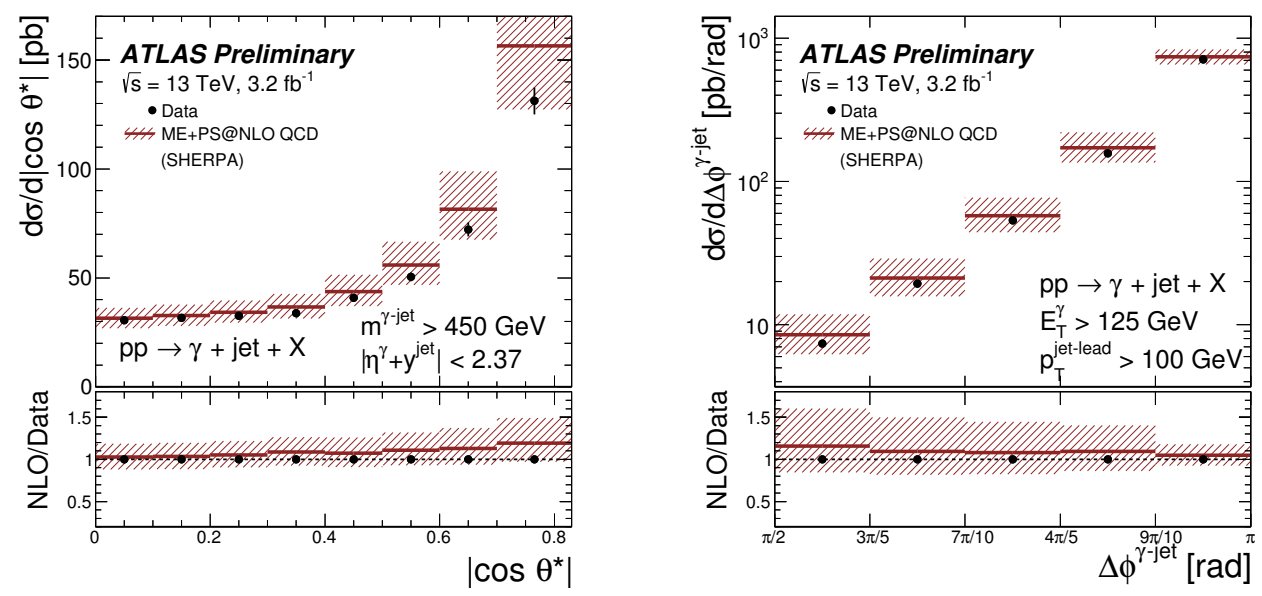

Figure 2: Measured cross sections for isolated-photon plus one-jet production (dots) as functions of $\left|\cos \theta^{*}\right|$ (left) and $\Delta \phi^{\gamma-\text { jet }}$ (right) . For comparison, the multi-leg NLO QCD plus parton shower predictions from Sherpa (solid lines) are also shown. The bottom part of each figure shows the ratios of the predictions to the measured cross section. Ref. [3]. 
The agreement for the fiducial cross section improves for fixed order calculation when moving from NLO to NNLO. Many differential cross sections are measured and the agreement improves when moving from NLO prediction to NNLO but in general fixed order predictions are unable to reproduce the data. In particular fixed order calculations are not expected to give reliable predictions in regions sensitive to infrared emissions. In this case the data are well reproduced by the inclusion of soft-gluon resummation at NNLL by Resbos (an example is shown in Figure 3).
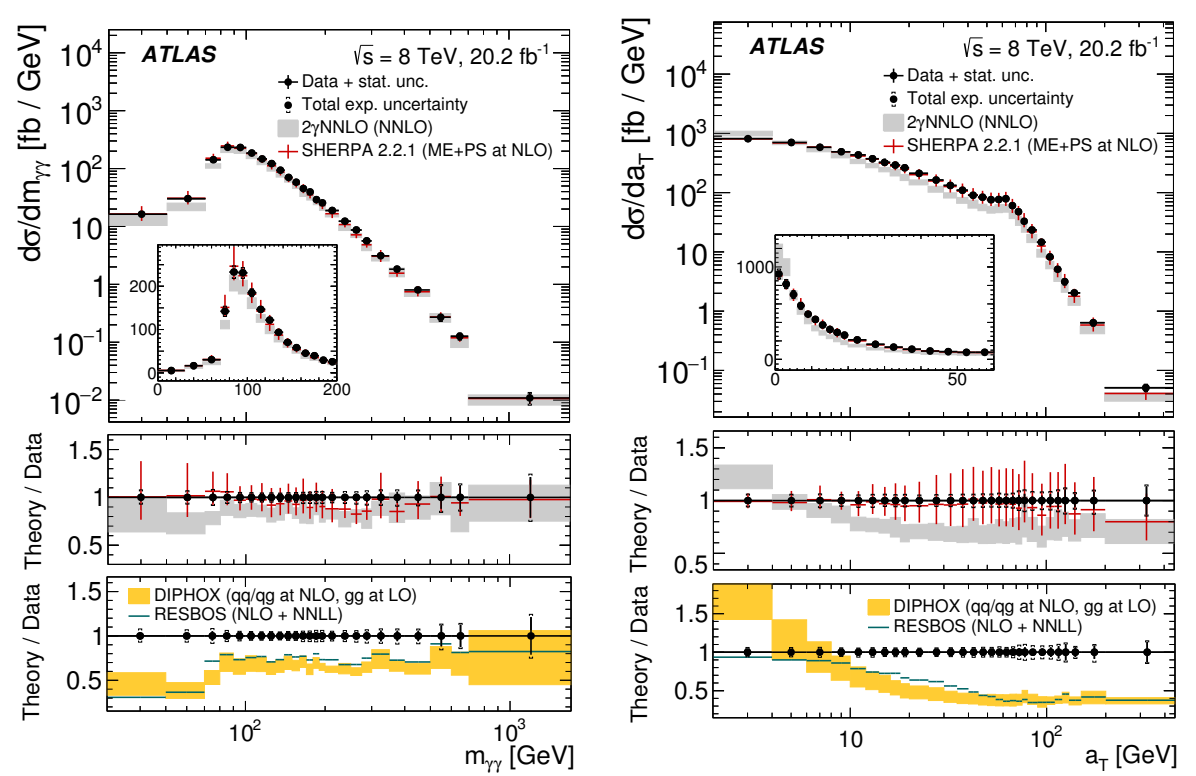

Figure 3: Differential cross sections as functions of (left) $m_{\gamma \gamma}$ and (right) $a_{T}$ compared to the predictions from Sherpa 2.2.1 and $2 \gamma$ NNLO. At the bottom of each plot, the ratio of the prediction to the data is shown. The ratio of Diphox and Resbos to the data is also shown. Ref. [5].

\section{References}

[1] ATLAS Collaboration, 2008 JINST 3 S08003

[2] ATLAS Collaboration, Phys. Lett. B 770 (2017) 473

[3] ATLAS Collaboration, ATLAS-CONF-2017-059, https://cds.cern.ch/record/2273875

[4] ATLAS Collaboration, Nucl. Phys. B 918 (2017) 257

[5] ATLAS Collaboration, Phys. Rev. D 95 (2017) no.11, 112005 